\title{
Design of a Portable Levitating Ball PID Control Trainer System and Curriculum for Electrical Engineering Technology Students
}

\section{Mr. Kevin Zender, Michigan Technological University}

Kevin is a recently graduated student from Michigan Technological university. He completed his undergrad in Electrical Engineering Technology with a minor in Data Acquisition and currently has a full time position at JR Automation as a Controls Engineer. Other interests include sustainability and woodworking.

Mr. Corey Blankenship, Michigan Technological University

Mr. Tyson Joseph Bethke, Michigan Technological University

Dr. Nathir A. Rawashdeh, Michigan Technological University

Nathir Ahmad Rawashdeh joined the College of Computing and Department of Applied Computing faculty in August 2019. Prior to this appointment, he was an associate professor in the Mechatronics Engineering Department at the German Jordanian University, where he spent 10 years. He served as the Acting Vice Dean of the School of Applied Technical Science, the Chair of the Department of Mechatronics Engineering, and the academic Exchange Coordinator with German partner universities. He spent two years as a Senior Software Engineer, in the laser division's Color Science and Imaging Department, at Lexmark International, Inc. in Lexington, Kentucky. Between 2000 and 2003, he was with The MathWorks, Inc. in Natick, Massachusetts working on software quality engineering for embedded DSP programming using MATLAB and Simulink. He has experience with European Union funded projects and served on several organizing and review committees. He is fluent in English, German and Arabic. His interests include unmanned ground vehicles, electromobility, robotics, image analysis, and color science. Dr. Rawashdeh is a Senior Member of the IEEE. 


\title{
Design of a Portable Levitating Ball PID Control Trainer System and Curriculum for Electrical Engineering Technology Students
}

\author{
Kevin Zender, Corey Blankenship, Tyson Bethke, Nathir Rawashdeh \\ Department of Applied Computing, Michigan Technological University, Houghton, MI
}

\begin{abstract}
This paper details the design of a levitating ball portable training system for in-depth learning of Proportional Integral Derivative (PID) control theory. This system can be incorporated into the Electrical Engineering Technology bachelor degree curriculum laboratories at our university. Based on the prevalence of PID control applications in industry, and it being a relatively advanced concept in traditional, theory heavy, control system courses, it is important to address this topic with a practical system. This has inspired the idea of designing a PID training lab course for the department. Focusing on analog circuits and programmable logic controllers, this lab course would give an in-depth look at the physical layout of the PID control system and how it operates. The laboratory procedures themselves include a physical demonstration of the software implementation of the PID calculation, with access to manipulate the code. PID tuning and understanding how gains at each stage affect the overall outcome of the control system response will be the highlights of the laboratory procedures. The overall layout of the design of this training lab could function as a framework curriculum, meaning that it could be used at other learning institutions other than our university. There are 3 existing courses within the Electrical Engineering Technology (EET) bachelor curriculum that have the flexibility to implement this form of training. This project looks to aid the preparation of future undergraduates interested in automatic control implementations.
\end{abstract}

\section{Introduction}

PID control is an integral component of industrial automation [1,2]. It is used to automatically control process variables such as temperature, liquid level, pressure, flow rates, etc. Although programmable logic controllers (PLCs) have software functions for implementing PID control using analog input and output modules, all major manufacturers offer stand-alone PID controllers since they are easier to program, monitor and maintain [3]. This creates a need to teach this cornerstone control calculation to university-level engineering students. The applicability of PID in many different career fields, implies that many degree programs could benefit from adding it to their curriculum. Mechanical Engineering, Electrical Engineering, Chemical Engineering, Electrical Engineering Technology, Mechanical Engineering Technology, etc. are all degrees that either already offer disciplines or courses that study the design and use of automated control [5]. 
Part of the Advanced PLC laboratory at our university, the Amatrol Process Control System, shown in Fig. 1, is used to teach programming of industrial equipment for controlling the flow rate and level of fluids [3]. It is not concerned with the theory of PID control, rather it focuses on the programming of industrial equipment and the application. A multitude of automatic control laboratory education kits exists[6]. These use various applications including process, temperature, motor speed, angle, altitude, and pressure control. These education systems are typically expensive, application-oriented, and proprietary. This paper outlines the design of a smaller, more easily replicable, PID demonstration unit, as well as curriculum for three short lab experiments to interact and learn with it. This will let the PID education fit into existing courses that cover automation control, and be more accessible to a larger number of students.

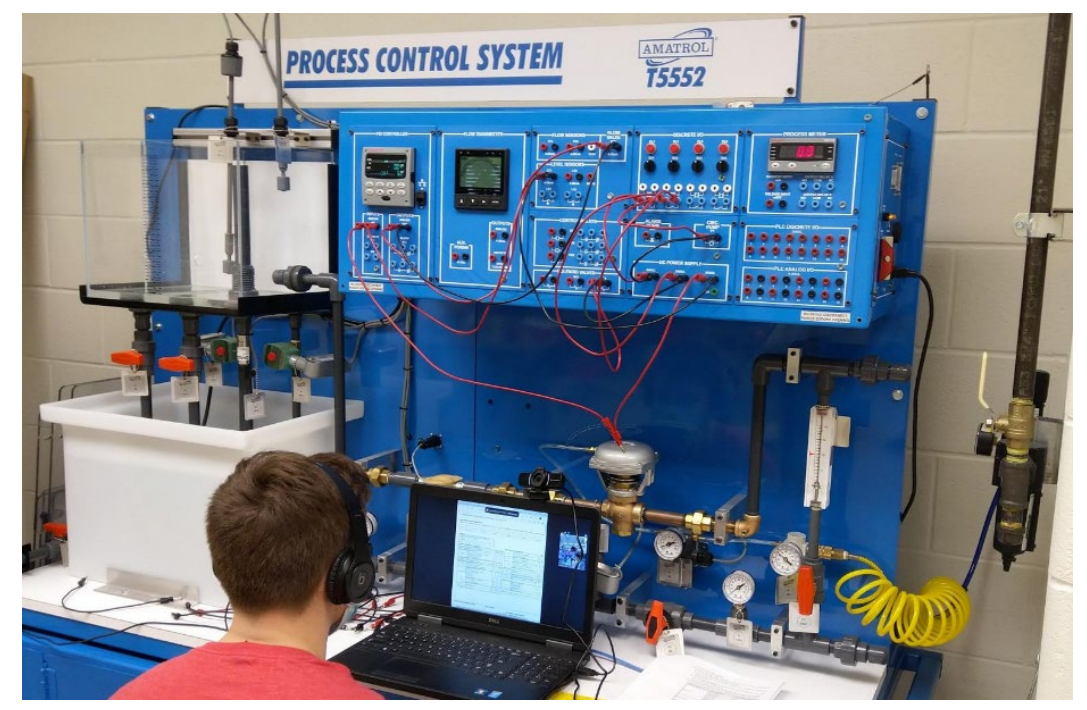

Figure 1. PID Process Control training in automation laboratory [3]

\section{PID Control Loop}

The developed trainer implements PID control of a ball's position, i.e. the process variable, in a vertical transparent tube, through a base mounted air fan as the actuator. The ball height is measured, i.e. fed back, and compared to the setpoint, that is the desired ball height. The PID gains can then be tuned to change the system time response to disturbance of the height, or changes in the height setpoint. Figure 2 illustrates the feedback control loop of the ball height PID control system trainer. The PID gains, $K p, K i$, and $K d$, respectively, are tunable via potentiometers by the student and their value displayed on the interactive system screen. The disturbance to the ball height is an additive or subtractive change to the ball height induced by restricting or increasing airflow, say by the student briefly placing their palm on top of the tube where the air is exhausted. 


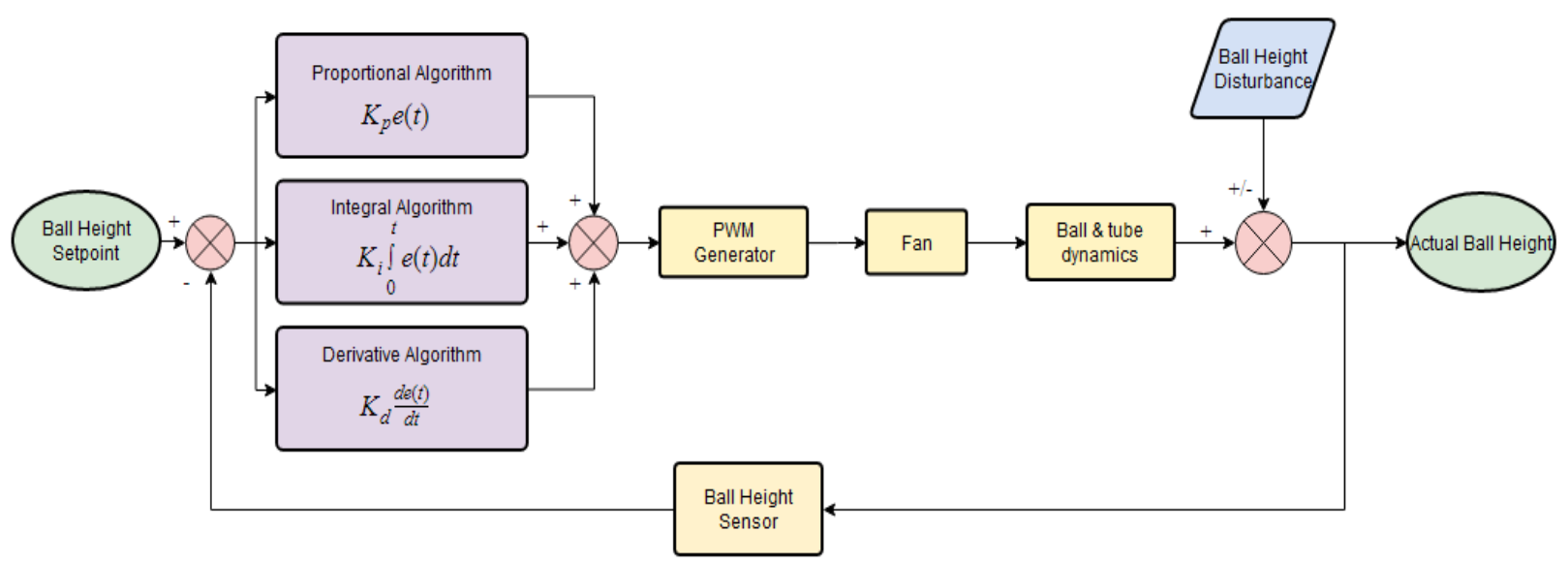

Figure 2. Feedback control loop of the ball-height controller system

The PID controller's output is used to control the fan speed through a Pulse Width Modulation (PWM) function, and is a function of the ball height error $e(t)$ and the PID gain, as shown in eq.(1):

$$
u(t)=K p \times e(t)+K i \times \int_{o}^{t} e(t) d t+K d \times \frac{d}{d t} e(t)
$$

The PID controller equation shows how the controller output, $u(t)$ is a weighted sum of the current error $e(t)$, as well as the accumulator error, and the change in error.

\section{Mechanical Design}

The trainer device is used as an interactive visual representation of Proportional, Integral and Derivative control schemes. A similar design was created at ETSI Informatica [7]. The ball inside a clear tube is used to demonstrate the controls effect on the system; as the ball moves further from the setpoint, the system will control the speed at which the fan spins, affecting the amount of air moving through the tube, which affects the position of the ball. Gravity acts as a constant downward force on the ball, and the fan is a variable control to create upwards force on the ball. Holes drilled into the cap of the vertical tube are used as interactive disturbance on the system.

The mechanical design seen in Fig. 3 was designed to fulfill the niche of this trainer device. The vertical cylinder was made from a 2" OD x 1/8" wall acrylic tube [8] and the base for the components and fan are constructed from 3/16" acrylic [9], which provides students with clear observation of the process for the educational benefit. The VL53L0X sensor [10] is mounted to a 3D printed cap on the top of the tube. The cap also includes drilled holes to allow for air to exit the chamber and simultaneously provides a manageable means to introduce outside disturbance to the system by covering the holes. The base mount for the fan [11] and tube is used to house 
the electrical components and control panel for interactive variable control. Potentiometers [12] and double pole - double throw switches [13] provide students with variable control, and a 4 inch display screen. Between these input and output components, the achieved design is a standalone unit, without the need for outside equipment.

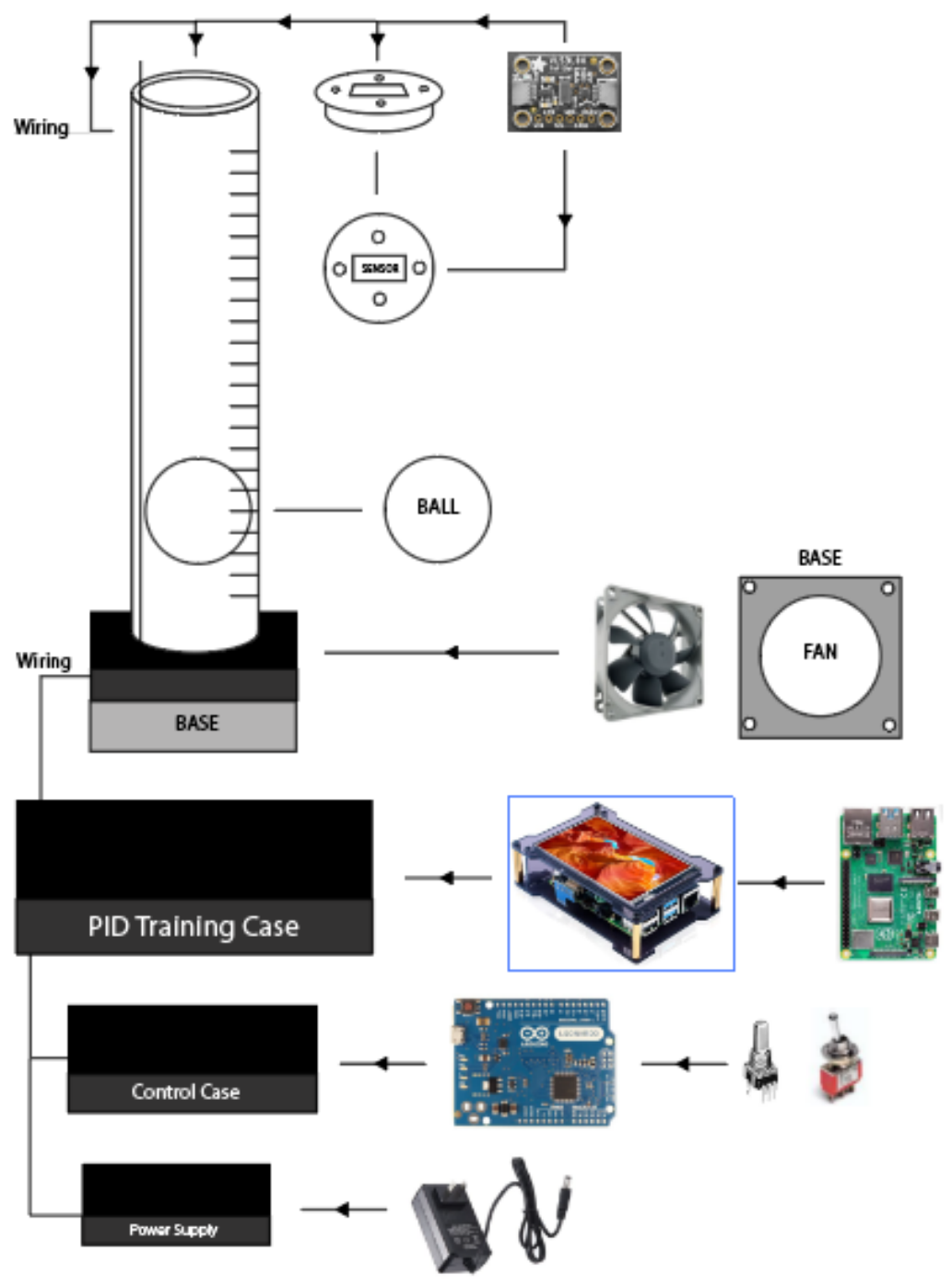

Figure 3. Mechanical components of the floating-ball trainer

\section{Electrical Design}

The trainer deceive has several requirements. Firstly, it must sense the position, i.e. height, of the ball in the cylinder. It must also actuate the fan through a PWM control signal, demonstrate on/off control, and calculate a PID gain control based on variable user inputs. As an added bonus 
it should be able to display important quantitative data to students in real-time, such as individual PID gains and the position of the ball in reference to time.

A circuit was designed to handle all of these functions. In Fig. 4, an Arduino Leonardo [14] receives an analog signal from the resistance potentiometers [12] for variable control, and discrete signals from throw switches [13] to achieve on/off control. The Arduino also receives a digital signal from our laser sensor [10] to identify ball position. The microcontroller also handles the PID calculation and converts the resultant into a PWM control signal for the fan [11]. A separate Raspberry Pi Microcontroller [15] receives serial communication from the Arduino in real time, and can plot relevant data to a screen for student observation.

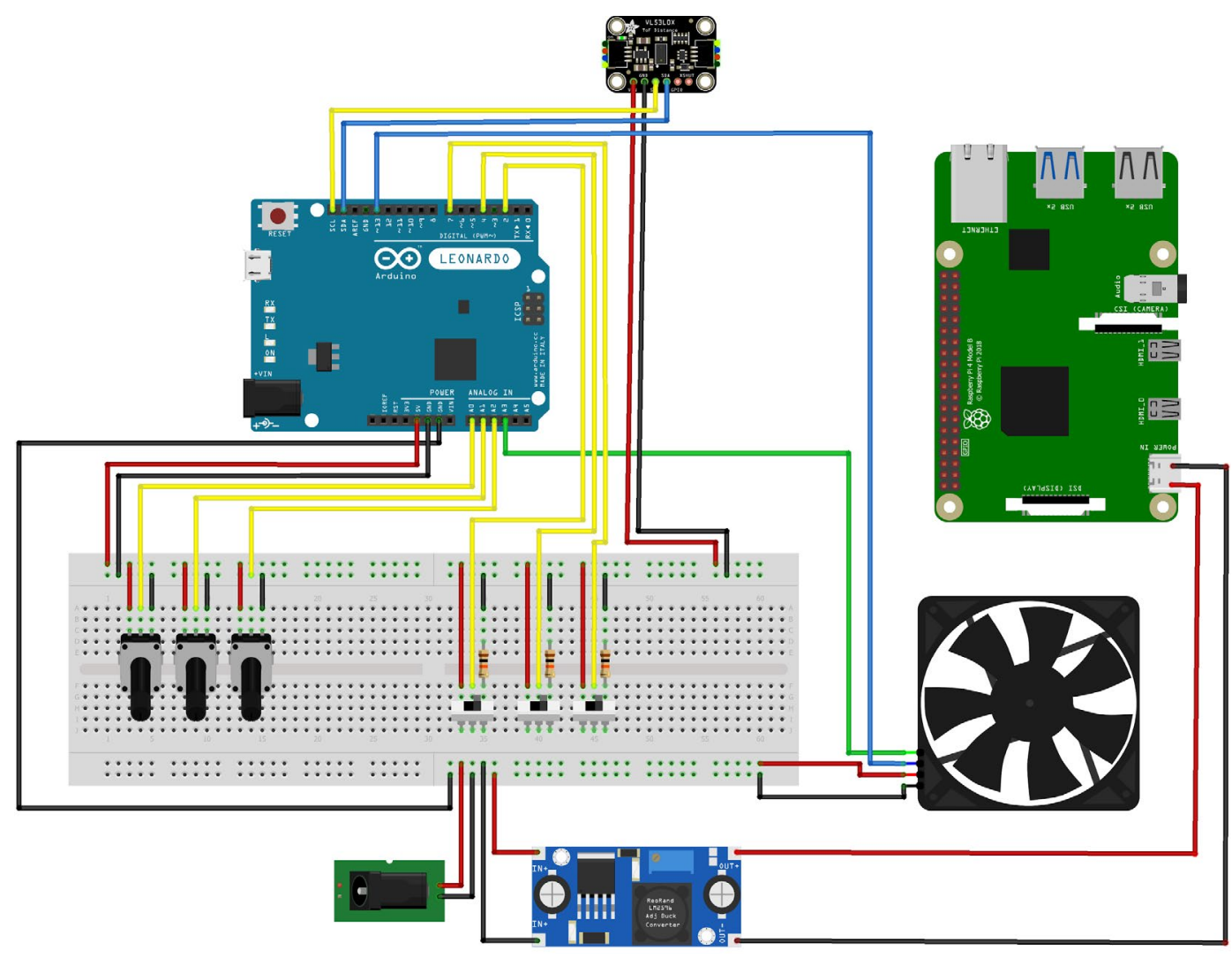

Figure 4. Electrical Circuit Diagram for the trainer.

\section{Software Design}

The program that was developed for the trainer utilizes different programming software across two microcontrollers. Most importantly, the Arduino Leonardo Microcontroller board [14] is used as the primary controller of the device, reading data from the sensor and user input components and sending a digital control signal to the fan [11]. Figure 5 describes the general execution of the developed code. 


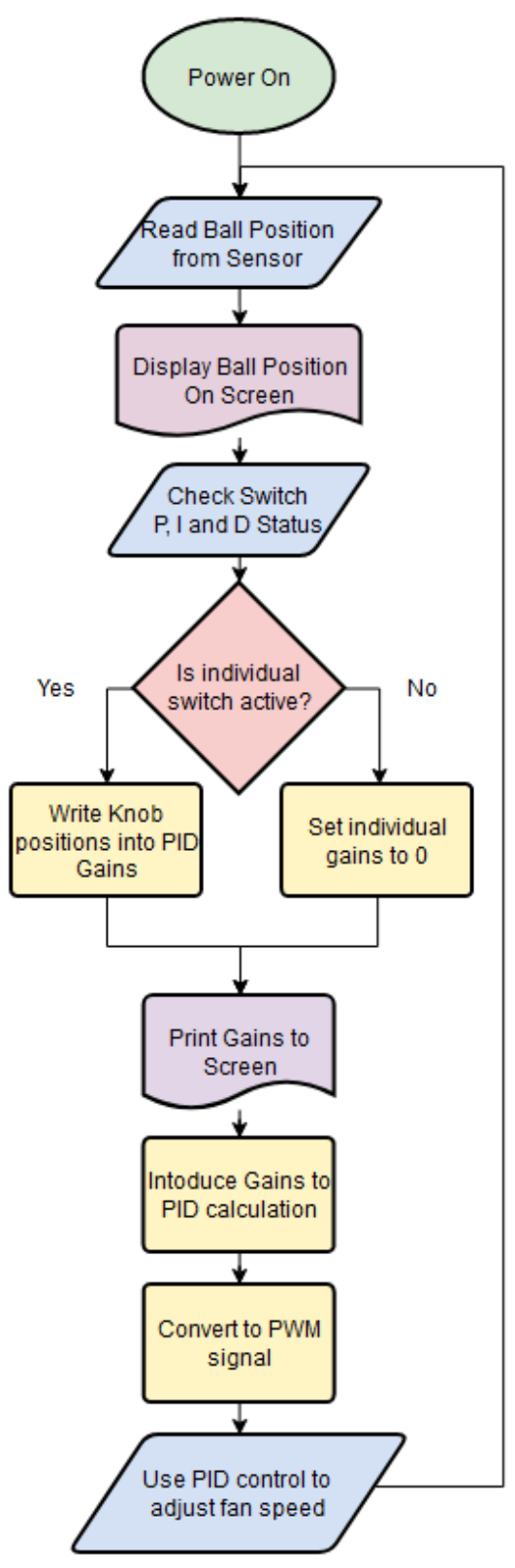

Figure 5. Microcontroller Program Flow Diagram

First the Arduino reads the ball's position from the VL53L0X sensor [10] and sends that data to the Raspberry Pi 4 4GB Model B [15] via a serial transmission. Then the Arduino reads the user $\mathrm{P}, \mathrm{I}$ and D status switches (each switch has corresponding on/off control over the corresponding PID gain)[13]. If the switch is high, the arduino reads the analog potentiometer value [12] and writes it into the corresponding gain value. If the switch is low, the arduino will overwrite the existing gain to 0 , removing that control ( $\mathrm{P}$, I or D) from the equation. Given a small $\mathrm{P}$ (proportional) gain value in the calculation, the system output response is minimal, thus it is used as an on/off control for power to the fan. After the gain for each expression has been calculated 
the Arduino sends the gain values to the Raspberry Pi as well. Then the Arduino calculates the PID sum value, and converts it to a PWM signal, which it uses to control the speed of the fan.

The Python code generated for the Raspberry Pi has one purpose, to display real time data to the user with the Muizie Raspberry Pi 4 touchscreen. The Pi charts the Ball position in real-time onto the displayed graph, and reads the $\mathrm{P}, \mathrm{I}$, and $\mathrm{D}$ gain values into the display as well. This gives the student access to real time data that is being calculated and then the immediate control feedback for that data.

\section{Curriculum Design}

To strengthen student understanding of PID and ensure the feasibility of the curriculum as a supplementary work to preexisting courses, the lab was written to be split into three separate sections. It was assumed that this lab would be taught after or during the explanation of PID in traditional resuscitation, so students should know of PID and its importance. This lab curriculum, then, exists to demonstrate to students some common understandings of the Fig. 5. flow diagram of PID control, such as the importance of each calculation, developed software and PI or PD control schemes.

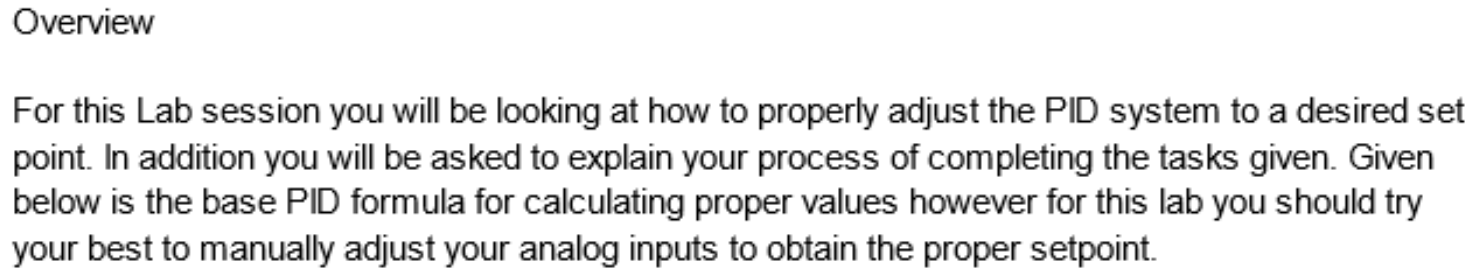

Figure 6. Overview section of Lab 1 for the proposed lab curriculum

The first section of the lab will introduce students to the problem and goal of the demonstration; the ball should be at the setpoint, and the most efficient way to keep it there respectively - as seen in Fig. 6. Students will be asked to use P alone to control the ball, and to identify its advantages compared to on/off control. Then students will be instructed to introduce Derivative control, and asked to demonstrate its use in the scope of the control scheme and identify specifically what has been achieved by this calculation. The conclusion of the first section introduces Integral control and its ability to reduce steady state error.

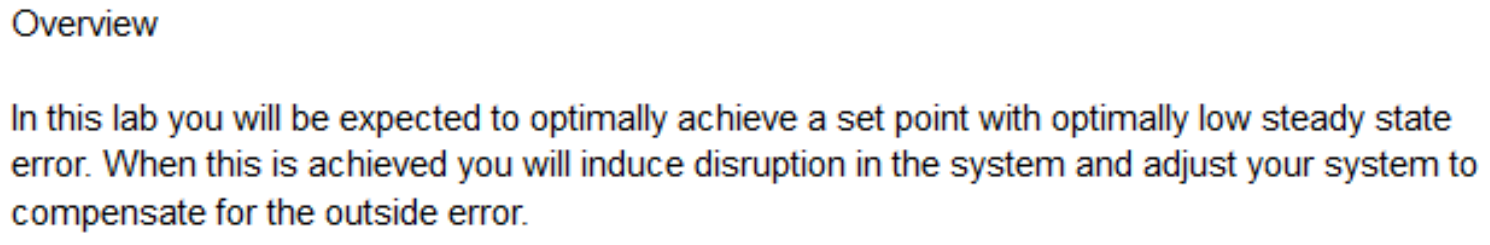

Figure 7. Overview section of Lab 2 for the proposed lab curriculum 
The second section of the lab, introduced in Fig. 7, will teach students how to use full PID control to make the most efficient control of the system and introduce outside disturbances. Students will be asked to use the different constants to create an equation to best fit given parameters such as necessary rise times. Students will be asked to create settings for the system to best deal with outside interference by covering the exhaust holes on the cap of the tube. This teaches them how you use the constants in conjunction with one another to form a steady system.

Overview

Using exclusively PI and PD control you will again be asked to complete scenarios to quantitatively evaluate the differences of PI and PD controls. Then you will be asked to qualitatively evaluate the differences between PI and PD.

Figure 8. Overview section of Lab 3 for the proposed lab curriculum

The third and final part of the lab section instructs students to use PI and PD. As the introduction states in Fig. 8, students will identify strengths, drawbacks, and uses for both PI and PD by observing firsthand the capabilities of the limited controls. Completion of this section shows that students understand the use of $\mathrm{P}, \mathrm{I}$ and $\mathrm{D}$ control and the benefits that each calculation brings to the summation.

\section{Conclusions}

The developed PID trainer unit is affordable, small, and simple to use. When paired with the proposed curriculum, the system will offer students a learning opportunity on a highly applicable and popular control scheme. The concise nature of the trainer allows several groups of students to have access to different units and allow them to focus on the core aspects of PID control. The fast-paced visuals of the mechanical system, as well as the real time data from the screen, allows for students to make several individual tests to affirm their understanding of the process. The software is developed as part of the trainer, so students don't need to spend time learning supplemental programming languages, and instructors can modify and fit this into several courses without constraint issues. Further study can be done on the curriculum to improve its performance. As students go through this program and use the trainer, professors can observe what about the curriculum is teaching as intended and what portions are maybe missing the mark. As changes in industry occur, the curriculum could be additionally changed to better suit modern developments. Additionally the Arduino microcontroller could be completely replaced with the Raspberry $\mathrm{Pi}$, as the $\mathrm{Pi}$ has all of the necessary electrical connections and the capability of generating a PWM signal. 


\section{References}

[1] V. Saravana and R. B. Kumar, "Design and Analysis of an Intelligent PID Controller for Sugar Industry Process," 2019 International Conference on Computational Intelligence and Knowledge Economy (ICCIKE), Dubai, United Arab Emirates, 2019, pp. 784-789, doi: 10.1109/ICCIKE47802.2019.9004285.

[2] W. Chen and X. Yang, "An Application of Adaptive Fuzzy PID Controller in Packing Industry," 2007 International Conference on Machine Learning and Cybernetics, Hong Kong, China, 2007, pp. 651-654, doi: 10.1109/ICMLC.2007.4370225.

[3] PID Controller Module, T5552-C1, Amatrol Inc., [Online]. Available: Amatrol, https://amatrol.com/coursepage, [Accessed January 28, 2021].

[4] Learning Systems for Industry 4.0, Festo Didactic, [Online]. Available: Festo-Didactic https://www.festo-didactic.com/int-en/learning-systems/, [Accessed March 6, 2021]

[5] E. I. Merzlikina and O. G. Prochina, "Laboratory Works on Control Theory Using Scilab/Xcos," 2020 V International Conference on Information Technologies in Engineering Education ( Inforino ), Moscow, Russia, 2020, pp. 1-4, doi: 10.1109/Inforino48376.2020.9111704.

[6] PID Loop Educational Tool, SAB3T, Hackaday.io, [Online] Available: Hackaday.io, https://hackaday.io/project/, [Accessed March 6, 2021].

[7] Chacon, Jesus, Jacobo Saenz, Luis De la Torre, Jose Manuel Diaz, and Francisco Esquembre. "Design of a low-cost air levitation system for teaching control engineering." Sensors 17, no. 10 (2017): 2321.

[8] Wall Clear Cast Acrylic Tubing, 2" OD x 1/8", United States Plastic Corp., [Online]: Available: USPC, https://www.usplastic.com/catalog, [Accessed March 6, 2021]

[9] Acrylic Extruded Clear Sheet, 3/16" 24"x48", AcmePlastics, [Online] Available: AcmePlastics.com, https://www.acmeplastics.com/acrylic-sheets, [Accessed March 6, 2021]

[10] Time of Flight Micro-LIDAR Distance Sensor, VL53L0X, Adafruit, [Online] Available: Adafruit, https://learn.adafruit.com, [Accessed: March 6, 2021]

[11] Noctua Cooling Fan, NF-R8 1800, Noctua, [Online] Available: https://noctua.at/en/nf-r8, [Accessed March 6 2021]

[12] Potentiometer, RK09L1140A65, Mouser Electronics [Online]. Available: Mouser Electronics, https://www.mouser.com, [Accessed March 6, 2021]

[13] Alcoswitch Gemini A Series, Toggle Switches, 2-1825138-8, Alcoswitch, [Online] Available: TE Connectivity, https://www.te.com/usa-en [Accessed March 6, 2021]

[14] Arduino Leonardo Micronctonroller, ATmega32u4, Arduino [Online] Available: Arduino, https://www.alliedelec.com, [Accessed March 6, 2021]

[15] Raspberry Pi, Raspberry Pi 4, Raspberry Pi, [Online] Available: Raspberry Pi, https://www.raspberrypi.org/products, [Accessed March 6, 2021] 